\title{
pilo, a gene required for glycosylation of Pseudomonas aeruginosa 1244 pilin
}

\author{
Peter Castric
}

Tel: +1 412396 6319. Fax: +1 412396 5907. e-mail: Castric@ DUQ3.CC.DUQ.EDU

Department of Biological

Sciences, Duquesne

University, Pittsburgh,

Pennsylvania 15282, USA

\begin{abstract}
Nucleotide sequencing of a region downstream from the Pseudomonas aeruginosa 1244 pilin structural gene, pilA, revealed an ORF potentially able to code for a protein of $M_{r} 50862$. This ORF, called pilO, was flanked by a tRNAthr gene, which was followed by a transcriptional termination sequence. The tRNA $^{\text {thr }}$ gene and the termination sequence were nearly identical to sequences found immediately adjacent to the pilA gene of several $P$. aeruginosa strains. A 2200 base mRNA strand, which contained both the pillo and pillA transcripts, was produced from this region, while a 650 base transcript containing only pilA was present in a 100-fold excess over the longer transcript. Hyperexpression of the pilA gene in a Pilo- strain resulted in normal pilusspecific phage sensitivity and twitching motility. The pilin produced by this strain had a lower apparent $\boldsymbol{M}_{\mathrm{r}}$ and a more neutral $\mathrm{pl}$ compared to that produced by a strain containing a functional pilo gene. This pilin failed to react with a sugar-specific reagent which recognized pilin produced by the strain containing a functional pilo gene.
\end{abstract}

Keywords: Pseudomonas aeruginosa, type 4 pili, protein glycosylation, pilus assembly, fimbriae

\section{INTRODUCTION}

Many Gram-negative bacteria produce thin extracellular protein filaments called pili. The Pseudomonas aeruginosa pili are similar in structure and function (Paranchych \& Frost, 1988) to those produced by species of the genera Dichelobacter (formerly Bacteroides; Elleman, 1988), Eikenella (Rao \& Progulske-Fox, 1993), Kingella (Weir \& Marrs, 1992), Moraxella (Marrs et al., 1985), Neisseria (Meyer et al., 1984) and Vibrio (Faast et al., 1989). These pili, which are all organized as bundles of 6-nm-diameter hollow fibres and which have the ability to extend and retract, are classified as type 4 (Ottow, 1975). The pili of $P$. aeruginosa are located at the cell's pole and function to facilitate adherence, a property important in virulence (Baker, 1993; Ramphal et al., 1984; Wood et al., 1980), a form of short-range motility called twitching (Bradley, 1980), and bacteriophage recognition (Bradley, 1972).

The $P$. aeruginosa pili are composed of a $15-16000 M_{\mathrm{r}}$ monomeric pilin subunit, which is arranged in a helical fashion within the pilus fibre (Watts et al., 1983). The first

The EMBL, GenBank, and DDBJ accession number for the nucleotide sequence data reported in this paper is X83916 PAPILAO.
30 amino acid residues of the pilin from this species are highly conserved, while the remainder vary with strain source. Characteristic variation among pilin primary structure allows differentiation of $P$. aeruginosa pilins into at least two groups (Castric \& Deal, 1994). This pilus grouping is further supported by nucleotide sequence homology of the regions flanking the pil $A$ gene. The region following the pil $A$ gene of $P$. aeruginosa strain 1244 (a group I strain) contains a ribosome-binding sequence and a start codon not seen in the group II strains. This paper presents evidence that this start codon is the beginning of an ORF, referred to as pilO. It is proposed that this gene codes for a protein which is involved in post-translational modification of pilin in which an acidic carbohydrate moiety is attached to the pilin protein.

\section{METHODS}

Bacterial strains and culture conditions. $P$. aeruginosa 1244 , a clinical isolate which has been used in pilus-mediated adhesion studies (Ramphal et al., 1984), was provided by A. T. McManus, US Army Institute of Surgical Research, San Antonio, Texas, USA. $P$. aeruginosa strain 1244 N3, a mutant which is unable to make pili or produce pilin (Ramphal et al., 1991) due to an inactivated $r$ o $N$ gene, was furnished by $\mathrm{S}$. Lory, University of 
Washington, USA. Cultures were grown on LB medium (Sambrook et al., 1989) at $37^{\circ} \mathrm{C}$. Antibiotic concentrations in selective media used for $P$. aeruginosa were as follows: carbenicillin $(\mathrm{Cb}), 500 \mu \mathrm{g} \mathrm{ml}^{-1}$; tetracycline ( $\left.\mathrm{Tc}\right), 50 \mu \mathrm{g} \mathrm{ml}^{-1}$. Antibiotics used with Escberichia coli were: ampicillin (Ap), $75 \mu \mathrm{g} \mathrm{ml}^{-1}$; spectinomycin (Sp), $25 \mu \mathrm{g} \mathrm{ml}^{-1}$; Tc, $10 \mu \mathrm{g} \mathrm{ml}^{-1}$.

Nucleotide sequencing. The Sanger dideoxy chain-termination method (Sanger et al., 1977) was used. The template was singlestranded M13 subclones of phage lambda or plasmid clones (Castric et al., 1989) containing DNA downstream from the pil $A$ gene. Synthetic primers, derived from known sequences, or universal primers were employed.

Plasmid constructs. The plasmid pPAC46, which contained both pilA and pilO, was constructed by ligating the SpbI$H$ indIII plasmid fragment containing pil $A$ and adjacent DNA (Fig. 3) of pPAC202 (Castric et al., 1989) to plasmid pUC18 digested with these same enzymes. The EcoRI-HindIII fragment of this construct, pPAC124, was ligated with the broad host range expression vector $\mathrm{pMMB66EH}\left(\mathrm{Ap}^{\mathrm{r}}\right.$ lacl $\mathrm{T}^{\mathrm{q}}$; Furste et al., 1986), which had been digested with the same restriction enzymes. Plasmid pPAC24, which contained pil $A$, but not pilO, was constructed by ligating the pPAC124 EcoRI-NheI fragment containing the pil $A$ gene with pUC19 digested with EcoRI and XbaI. The EcoRI-HindIII fragment of this construct, which contained only the pilA gene, was ligated with pMMB66EH digested with the same restriction enzymes. The pil $A$ gene of both pPAC24 and pPAC46 was under control of the vector tac promoter. Since the $S p B \mathrm{I}$ site used in plasmid construction is located within the pil $A$ promoter neither pPAC24 nor pPAC46 were able to express the pilin structural gene using the $P$. aeruginosa promoter. Plasmid DNA was introduced into $E$. coli by transformation (Sambrook et al., 1989) and was transferred to $P$. aeruginosa by triparental mating (Ruvkun \& Ausubel, 1981).

Northern blot analysis. RNA was extracted as described by Reddy (1989) and separated by formaldehyde-agarose gel electrophoresis, stained with ethidium bromide, and transferred to nitrocellulose paper by capillary blot as described by Sambrook et al. (1989). Probe DNA, isolated from pPAC24 or pPAC46, was labelled by nick translation using $\left[\alpha-{ }^{32} \mathrm{P}\right] \mathrm{dCTP}$. Prehybridization, hybridization, and washing steps were as described by Sambrook et al. (1989). Detection of hybridization was by autoradiography. Densitometric scanning was carried out using an LKB Ultroscan laser densitometer.

Purification of pili. Production of pili by expression of the pil $A$ genes of plasmids pPAC24 and PPAC46 in P. aeruginosa 1244N3 was carried out using LB-agar- or LB-broth-grown cultures in the presence of $5 \mathrm{mM}$ IPTG. LB-agar-grown cells were resuspended in LB broth. These cells (as well as broth-grown cells) were depiliated by four excursions through a 22-gauge needle. Cells were removed by centrifugation ( $7500 \mathrm{~g}$ for $15 \mathrm{~min})$. The pili remaining in the supernatant fluid were purified by a variation of the method of Silipigni-Fusco (1987). In this procedure the supernatant fluid was made $0 \cdot 1 \mathrm{M}$ with respect to $\mathrm{MgCl}_{2}$, and stored on ice for $60 \mathrm{~min}$. The resulting precipitate, which contained the pilus fibres, was removed by centrifugation at $12000 \mathrm{~g}$ for $15 \mathrm{~min}$.

Pilin analysis. N-terminal amino acid sequence analysis and amino acid analysis was performed on purified pilin from strain 1244N3(pPAC24). This protein was subjected to PAGE (15\% gel), which was followed by electroblotting to polyvinylidene difluoride paper. Preliminary staining showed that pilin was the major protein present and that it was well separated from the minor contaminants present. Pilin bands were excised and subjected to gas-phase sequencing and to amino acid analysis (University of Pittsburgh Peptide Facility).
Pilin immunoblot analysis. Pilins were separated by PAGE $\left(12.5 \%\right.$ gel in the presence of either $0.1 \% \mathrm{SDS}$ at $5^{\circ} \mathrm{C}$ or $30 \mathrm{mM}$ octyl glucoside at $15^{\circ} \mathrm{C}$ ) or subjected to isoelectric focusing (pH 3.0-9.5) in the presence of $30 \mathrm{mM}$ octyl glucoside at $15^{\circ} \mathrm{C}$ using the LKB-Pharmacia Phastsystem. Proteins were transferred to nitrocellulose paper by diffusion blotting; pilin adsorbed to the paper was detected using anti-pilin monoclonal antibody 6-45 (Saiman et al., 1989) as previously described (Castric et al., 1989). The presence of pilin-bound sugar residues was detected by first derivatizing purified pili using the DIG Glycan Detection kit of Boehringer Mannheim Biochemica. This protein was subjected to SDS-PAGE (12.5\% gel) using the Bio-Rad Mini-Protean II system, in which temperature was neither controlled nor monitored. Pilin was electroblotted to nitrocellulose paper, probed with an alkaline-phosphataselabelled antibody specific for the hapten used in pilin derivatization, and developed as described by Boehringer Mannheim Biochemica.

Electron microscopy. Cells to be analysed by electron microscopy were grown on LB agar plates at $37^{\circ} \mathrm{C}$ for approximately $18 \mathrm{~h}$ and suspended in PBS $(0.145 \mathrm{M} \mathrm{NaCl}, 0.15 \mathrm{M}$ sodium phosphate). The cells were coated on 200 mesh formvarcoated copper grids, subjected to staining with $1 \%(\mathrm{w} / \mathrm{v})$ uranyl acetate, and viewed in a Philips 201 electron microscope.

Pili functionality tests. The phage sensitivity test was performed by streaking a loop of pilus-specific phage PE69 on an LB agar plate. This was cross-streaked with diluted $P$. aeruginosa 1244 strains to be tested. Phage sensitivity was interpreted from the absence of growth at the cross-streak junction. Twitching (Henrichsen, 1983) was determined by streaking strains of interest on a well-dried LB agar plate where motility was scored as spreading growth at between 48 and $72 \mathrm{~h}$ at $37^{\circ} \mathrm{C}$.

\section{RESULTS}

\section{Nucleotide sequence analysis}

The nucleotide sequence downstream from the $P$. aeruginosa strain 1244 pil $A$ gene (Fig. 1) contains a potential transcriptional termination structure situated between positions 626 and 708 (noted previously by Castric et al., 1989). A ribosome-binding site (GGAG) is seen at position 717 followed closely by three start codons, the first of which is located immediately in frame with a stop codon (TGA). The second and third, GTG and ATG, are in frame with each other and begin an ORF (referred to hereafter as pilO) which extends $1383 \mathrm{bp}$, using the ATG codon, to position 2114. Codon usage of the 461 codons of the pilO gene is consistent with that of most other $P$. aeruginosa genes (West \& Iglewski, 1988). However, significant differences are seen when it is compared with the adjacent $p i l A$ gene or with the pil $A$ genes of other $P$. aeruginosa strains (West \& Iglewski, 1988; Castric \& Deal, 1994). While the Phe codons, TTT and TTC, have frequencies of $20 / 29$ and $9 / 29$, respectively, in the pil $A$ gene, these codons occur at rates of $6 / 25$ and $19 / 25$, respectively, in the pilO gene. The Arg codons, CGT and GCG, appear at rates of $26 / 31$ and $2 / 31$ in the pil $A$ gene, but occur at rates of $7 / 31$ and $11 / 31$, respectively, in the pilO gene. In addition, the $\mathrm{G}+\mathrm{C} \%$ content of pilO $(60.1 \%)$ is consistent with the general range given for $P$. aeruginosa chromosomal DNA (West \& Iglewski, 1988), but quite different from the value of $52.2 \%$ determined for the strain 1244 pil $A$ gene. A search of the GenBank 


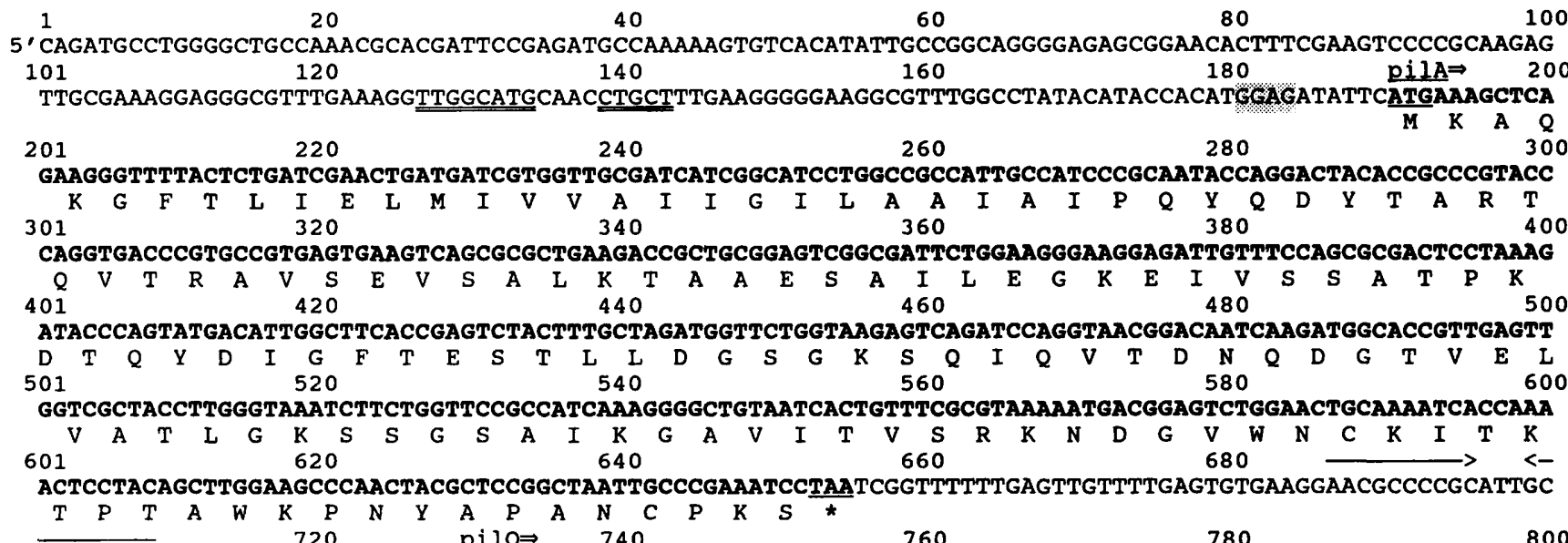
GGGGCGTTTTCATTCTGGAGCCCATGTGATGCGAATTTGGCTAGCCTGGGAAAGGATGGGTAGGGCGTCGCGTACTATTCTATTATTGCTGATAAGTATT $\begin{array}{rllllllllllllllllllllllllll}801 & & \text { M } & \text { R } & \text { I } & \text { W } & \text { L } & \text { A } & \text { W } & \text { E } & \text { R } & \text { M } & \mathbf{G} & \text { R } & \text { A } & \text { S } & \text { R } & \text { T } & \text { I } & \text { L } & \text { L } & \text { L } & \text { L } & \text { I } & \text { S } & \text { I }\end{array}$ CTTCTGCTTTCTCCCGTTGTTTACTGCGGGGTTTCCAAGAACTGGCATGATCAGCAGCGAATACTCCAATTGGTGGTGTTGAGTGGATCGTCACTCCTGC $\begin{array}{lllllllllllllllllllllllllllllllll}L & L & L & S & P & V & V & Y & C & G & V & S & K & N & W & H & D & Q & Q & R & I & L & Q & L & V & V & L & S & G & S & S & L & L\end{array}$ $\begin{array}{lllllllll}901 & 920 & 940 & 960 & 980 & 1000\end{array}$ TGCTCTTTTCTTCTCGTCTGTCATTTGCCAGAAGGATGGTTCAGGTAACACTPTTGGTTATCTTGGGTCTAGGCAGCGTTTCCGCATTTCTCTCCGCCAA

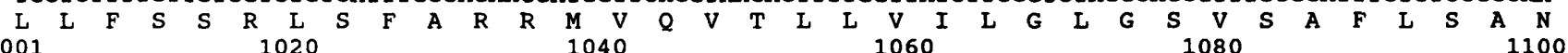
TCCTTCCTGGGCATTCAAGGAATGGTCGGTATTCGCCGGCCTGATGCTGTTTTCCTTCAATATATCCGCCAGCCCAGAGTGGGTTCGCCGTATCGCCCTC $\begin{array}{llllllllllllllllllllllllllllllllll}\text { P } & S & W & A & F & K & E & W & S & V & F & A & G & L & M & L & F & S & F & N & I & S & A & S & P & E & W & V & R & R & I & A & L\end{array}$

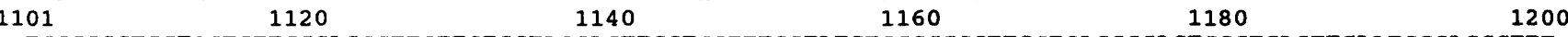
TGGGGGGTGGTCGTGTTGGGAGGGTTCTTCTGCTACCAGTTCCTGCTTTCCTATCTCGCGGCCTTCGTCAGCGGACTCCGTGAGTTGAATCCCAGGGTTT

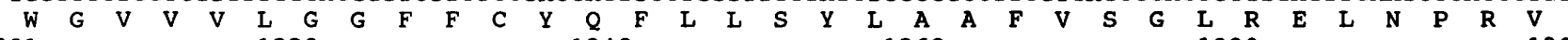

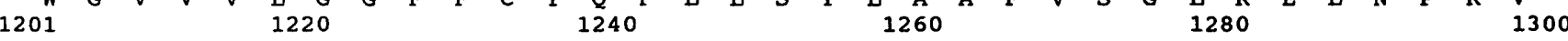
TGCTCAGCGGCTITTCCAACGTCCGGACCATGGGGCAGTTCCAGGCCATGCTGCTGCCGCTGATGGCTGCGCTGGGGTTGTACCTACGAGAGACCGGGCG


GTTCAGACTGTCCTGGCTTGTCATGCTGCTGCTGGCCATCCAATGGTGCATCTCCTTCGCCTTGGCTGGACGCGGGCTCTGGTTGGGTTTCGCCGTCGCG

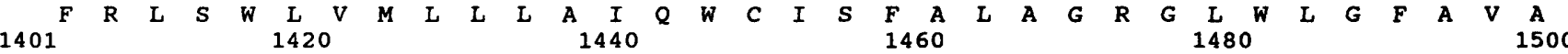
CATCTCGCGCTTTGCTGGATCGGCCCCTGGGGCGCCGCTTTTTGATCGTCCAGTTGTCCGCGGCATTCGTCGGGTGGCGCTCTATTTCCTGTPAATGG

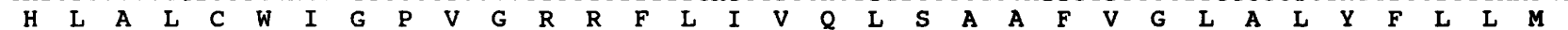

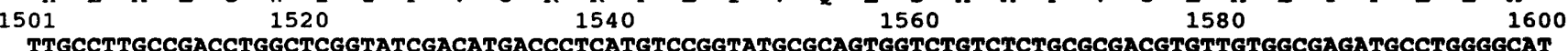
TTGCCTTGCCGACCTGGCTCGGTATCGACATGACCCTCATGTCCGGTATGCGCAGTGGTCTGTCTCTGCGCGACGTGTTGTGGCGAGATGCCTGGGGCAT

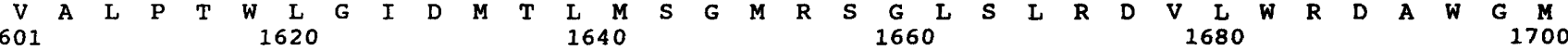
GTTCGTAGCCCATCCTTTGCTTGGGGTCGGGCCCATGCATTTCTCGGCGGTGCCGAACAGCGTCGGTGCCCACCCGCACCAGATGCTGCTGCAGTGGTTC

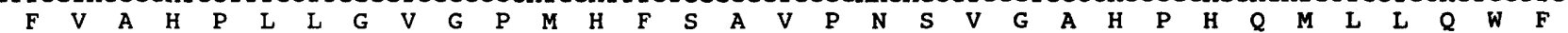

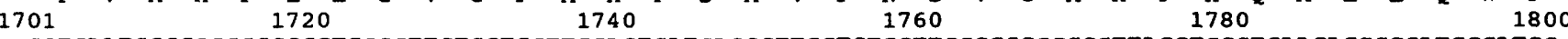
GCTGAATGGGGCGGGCCGCTGGCCTTCTGGTGGTTGGACTGATGACCCTTGGTCTGCTTCGCGGCGCGCGTTACCTGCGTGAACAGGGCGATCCGATGG

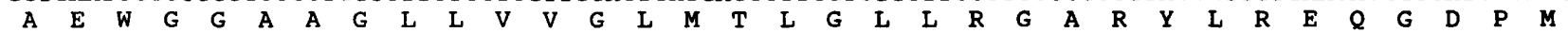

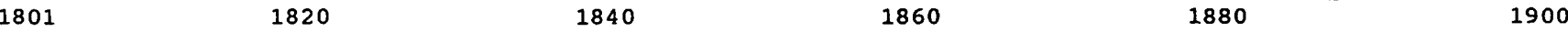
ACGCCGGACTGTGGCTGGCCCTCGTTTCGGTCCTGGTCTTGGCCCAGGTGGACGGCGTGTTCGTCATGCCCTTCACCCAGACCGTATTGGCCTTGCTGGT $\begin{array}{lllllllllllllllllllllllllllllllllll}\text { D } & \text { A } & G & \text { L } & \text { W } & \text { L } & \text { A } & \text { L } & \text { V } & \text { S } & \text { V } & \text { L } & \text { V } & \text { L } & \text { A } & \text { Q } & \text { V } & \text { D } & \text { G } & \text { V } & \text { F } & \text { V } & \text { M } & \text { P } & \text { F } & \text { T } & \text { Q } & \text { T } & \text { V } & \text { L } & \text { A } & \text { L } & \text { L } & \text { V }\end{array}$

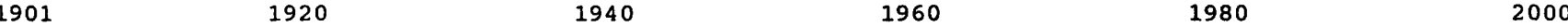
AGGCATCGCCATGGCGCGCTGGTCGAAGCCGGTCGTGCCGTCCCCGCACAGCGCTGGCTCTGTCGGGGCCTGGCTGTCGTTGTCATTGTCGTGCTGGGG

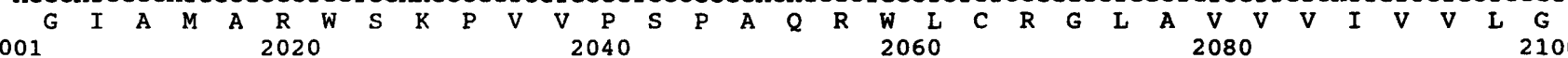
CGCGTGCTGCTGCTCGAGGTGCCGGGGCTGACCGCGGCCGAGGAGCGCTACCTGGAAATCCACGGCGGCGGTGAGGCGCCACGTTTCTGGATTCAGGGTT $\begin{array}{lllllllllllllllllllllllllllllllll}R & V & \text { L } & \text { L } & \text { L } & \text { E } & \text { V } & \text { P } & \text { G } & \text { L } & \text { T } & \text { A } & \text { A } & \text { E } & \text { E } & \text { R } & \text { Y } & \text { L } & \text { E } & \text { I } & \text { H } & \text { G } & \text { G } & G & \text { E } & \text { A } & \text { P } & R & F & \text { W } & \text { I } & Q & G\end{array}$ $210122120 \quad 2140 \quad 2160 \quad$ tRNA thr $\Rightarrow \quad 2180 \quad 2200$ GGATTCCCATGTGAGGGGATATCGGTTGAAATCCGGCTGTGCCGAGTGATACCTTCCCCGCGCCGCCGGATTAGCTCAGTCGGTAGAGCAGCTCATTCG W I P $M$ *

$220122202240 \longrightarrow 2300$ TAATGAGAAGGTCGGGGGTTCGATTCCTCTATCCGGCACCAGTCGCAATAAAAAGCCCCGCTTCGGCGGGGCTTTTTATTGCCTGCGATTCGTTCAGAGG


GTGAGGCGCATGGACAGGTCGACGGCCCTGACATCCTTGGTCAGGGTGCCGATGGAGATGTAGTCGACGCCGGTCTCGGCGATGTTGCGCAGGGTGCCCT 2401 2420

CGTTGATCCGCCGGAAGCTT 3 '

Fig. 1. Nucleotide sequence of the $P$. aeruginosa DNA containing the pilA gene and adjacent downstream region. The pilA region has been described previously (Castric et al., 1989). Bold-face nucleotide sequence indicates actual or proposed coding regions. Protein primary structure is shown beneath the coding regions. Single underlines denote start or stop codon positions, with an asterisk beneath a stop codon. Highlighted sequences are likely ribosome-binding sites. The double-underlined sequence is a proposed promoter region. Regions containing significant dyad symmetry are indicated by opposing arrows. 


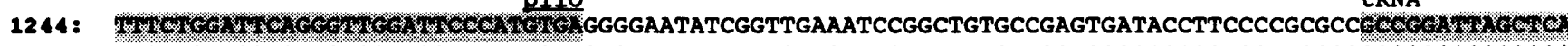

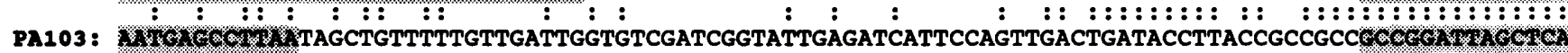
pilis

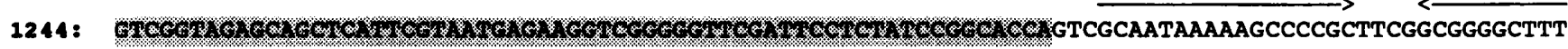

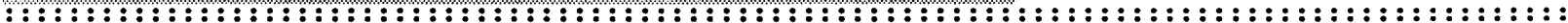

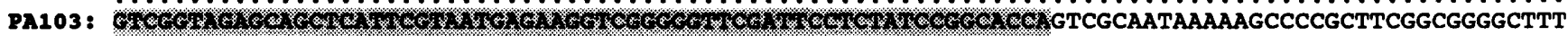

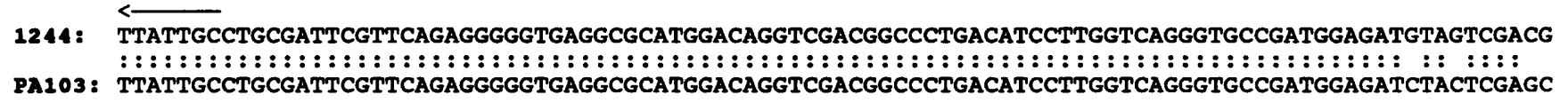

124: CCGGTCTCGGCGATGTTGCGCAGGGGTGCCCTCGTTGATCC-GCCGGAAGCTT

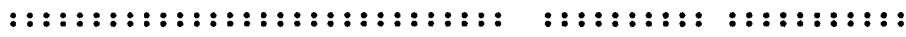

PA103: CCGGTCTCGGCGATGTTGCGCAGGGGGTGCTTTCGTTGATCCCGCCGGAAGCTT

Fig. 2. Sequence homology of the region downstream from the pilO gene, presented in Fig. 1, with the region downstream of the pilA gene of $P$. aeruginosa strain PA103 (Johnson et al., 1986). Colons indicate base homology. Regions containing significant dyad symmetry are indicated by opposing arrows. The tRNA gene region is highlighted.

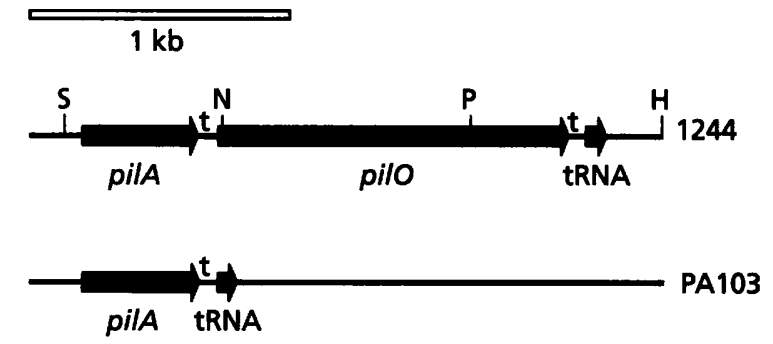

Fig. 3. Comparison of the pilin gene regions of $P$. aeruginosa strain 1244 and strain PA103. Potential transcriptional stops are indicated by the letter ' $t$ '. Restriction endonuclease sites: $H$, HindIII; N, Nhel; P, Pstl; S, Sphl.

database revealed no sequences with significant homology to the pilO gene.

A tRNA $^{\text {thr }}$ gene, which contains an anticodon recognizing the rare codon ACG, can be seen just downstream of the pilO gene between positions 2166 and 2241, and is followed, between positions 2245 and 2282, by a potentially bidirectional transcriptional stop containing a $17 \mathrm{bp}$ loop stem. A tRNA gene and transcriptional stop region have been noted previously (Hobbs et al., 1988) as occurring immediately downstream from the $P$. aeruginosa strain PAK pil $A$ gene, and may also be seen in the analogous region of the pil $A$ genes of $P$. aeruginosa strains PAO and PA103 (Johnson et al., 1986). A homology comparison (Fig. 2) shows that the tRNA transcriptional stop region described in this paper is virtually identical to the pil $A$ proximal regions of strain PA103 beginning at position 2421 and continuing 275 bp to the end of the sequence. It is interesting to note that the first five bases of this region (GAGTG) are also found, as a direct repeat, in the pil $A-O$ intragenic region beginning at position 676, suggesting a possible site of genetic recombination. These results are summarized in Fig. 3 where the organization of these regions may be compared.

\section{Transcription of pilo}

Northern blots (Fig. 4) of strain $1244 \mathrm{mRNA}$ showed that probe 1, composed of pilA DNA, recognized 650 base (RNA 2) and 2200 base (RNA 1) species. The smaller transcript was present in approximately a 100 -fold excess over the larger mRNA as determined by densitometric scanning. By analogy with work on other $P$. aeruginosa pil A genes (Johnson et al., 1986) the transcription startpoint may be presumed to begin between positions 150 and 160 (Fig. 1), suggesting that the larger transcript extends into the pilO region. The intermediate-sized RNA fragments might be formed by early termination, due to the loop structure referred to earlier, or might be degradation products of the larger species. Probe 2, which was composed of pilO DNA, hybridized only with the larger fragment and the intermediate pieces suggesting that the origin of the 2200 base mRNA is in the pil $A$ region and that synthesis of this molecule extends downstream through the pilO gene. This was confirmed by the reaction of probe 3 , which was composed of DNA from the latter part of the pilO gene and the tRNA transcriptional stop region. This probe hybridized with the larger fragment but with neither the pil $A$ transcript nor the intermediate fragments. This probe also hybridized with a very small RNA population, presumably tRNA produced by the pilO-associated gene, as well as with cross-reacting species. Overall these results indicate that pil $A, p i l O$ and probably the adjacent tRNA gene are part of a single transcriptional unit which utilizes the pil $A$ promoter.

\section{Influence of pilO on pilus function}

Since both pil $A$ and pilO are part of the same transcriptional unit, the inactivation of the pil $A$ promoter will also eliminate pilO expression. $P$. aeruginosa strain $1244 \mathrm{~N} 3$ is a mutant which is unable to make pili or produce pilin (Ramphal et al., 1991) due to an inactivated $r p o N$ gene. This strain was used as the genetic background for 
(a)

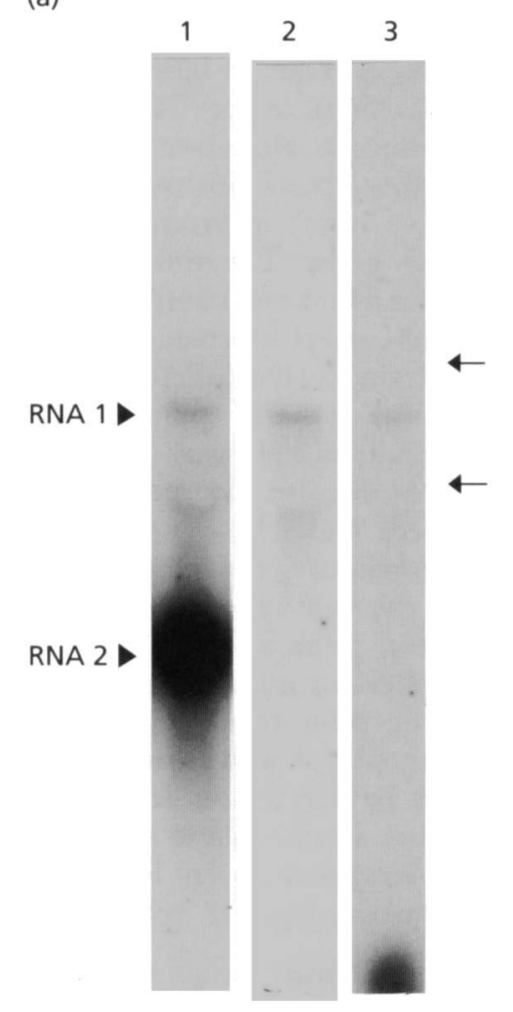

(b)

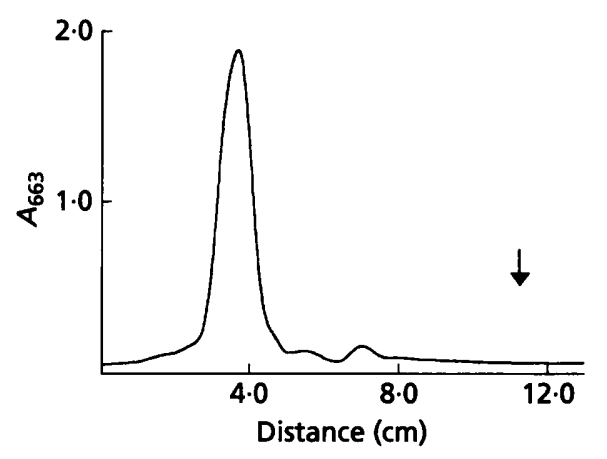

Fig. 4. Northern blot analysis of mRNA extracted from $P$. aeruginosa strain 1244. (a) Probes were prepared from restriction fragments of cloned DNA indicated in the Fig. 3 map as follows. Lanes: 1, probe 1 (the Sphl-Nhel fragment); 2 , probe 2 (the Nhel-Pstl fragment); 3, probe 3 (the Pstl-HindIII fragment). Arrows indicate positions of E. coli strain HB101 ribosomal RNA. (b) Densitometric scan of lane 1 of part (a) of this figure. Scanning was from bottom to top of the autoradiogram shown. The electrophoresis point of origin is marked by the arrow.

expression of the pil $A$ gene, carried on recombinant plasmids pPAC24 (containing only pil $A$ ) or pPAC46 (bearing both pil $A$ and pilO), under control of a tac promoter. Electron microscopy showed that while strain $1244 \mathrm{~N} 3$ produced no pili both derivatives 1244N3(pPAC24) and 1244N3(pPAC46), when grown in the presence of IPTG, produced pilus fibres which were indistinguishable from those of the wild-type (results not (a)

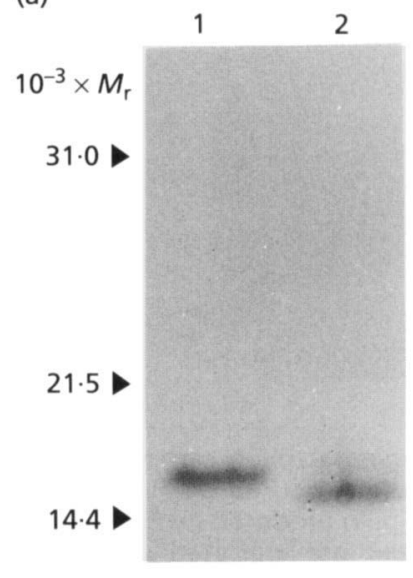

(b)

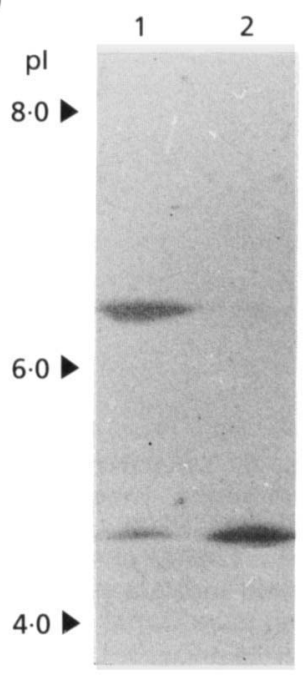

Fig. 5. Immunodetection of electrophoretically separated $P$. aeruginosa pilin. (a) SDS-PAGE of pilin at $5^{\circ} \mathrm{C}$. Lanes: $1,3 \mu \mathrm{g}$ pilin produced by $P$. aeruginosa strain 1244N3(pPAC46); $2,3 \mu \mathrm{g}$ pilin produced by $P$. aeruginosa strain 1244N3(pPAC24). $M_{r}$ values are indicated on the left. (b) Isoelectric focusing of pilin at $15^{\circ} \mathrm{C}$. Lanes: $1,3 \mu \mathrm{g}$ pilin produced by $P$. aeruginosa strain 1244N3(pPAC24); $2,3 \mu \mathrm{g}$ pilin produced by $P$. aeruginosa strain 1244N3(pPAC46). The $\mathrm{pH}$ gradient was determined by focusing pl standards on an identical gel in the absence of octyl glucoside.

shown). Both strains 1244N3(pPAC24) and 1244N3(pPAC46), in the presence of IPTG, were sensitive to the pilus-specific bacteriophage PE69 and demonstrated twitching motility on dried agar plates, properties lacking in strain 1244 N3. These results indicated that the absence of pilO did not appear to influence the ability of the pilus to extend and retract, qualities necessary for twitching and for phage sensitivity.

\section{Influence of pilO on pilin structure}

Although strain $1244 \mathrm{~N} 3$ was unable to produce pilin, as determined by Western blot analysis (results not shown), complementation of this strain with either plasmid pPAC24 or pPAC46, under inducing conditions, allowed production of this protein (Fig. 5a). While the $M_{\mathrm{r}}$ of mature pilin of strain 1244 predicted from the nucleotide sequence of the pil $A$ gene (Castric et al., 1989) is 15653, the apparent size of pilin produced by strain 1244 N3(pPAC46) or by wild-type strain 1244 (results not shown) is approximately 16900 . Pilin produced by strain 1244N3(pPAC24), which lacks a functional pilO gene, had an apparent $M_{\mathrm{r}}$ of 15600 . Identical results were obtained when gradient SDS-PAGE was employed. A small fraction of the pilin produced by strain 1244N3(pPAC24) migrated with wild-type pilin when the Bio-Rad MiniProtean II PAGE system (15\% gel) was employed (results not shown).

To explore the significance of this apparent $M_{\mathrm{r}}$ difference it was necessary to see if the $\mathrm{N}$-terminus of pilin produced 


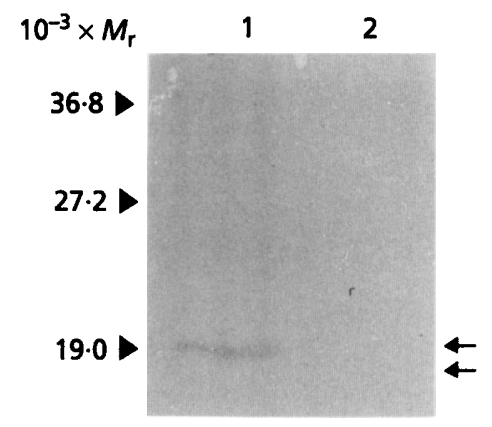

Fig. 6. Detection of $P$. aeruginosa pilin carbohydrate by immunoblot. Lanes: $1,10 \mu \mathrm{g}$ pilin produced by $P$. aeruginosa strain 1244N3(pPAC46); $2,10 \mu \mathrm{g}$ pilin produced by $P$. aeruginosa strain 1244N3(pPAC24). $M_{r}$ values are indicated on the left. Arrows indicate positions of these pilins on an identical blot using monoclonal antibody 6-45 for detection.

by strain $1244 \mathrm{~N} 3$ (pPAC24) was in any way altered. When this protein was subjected to $\mathrm{N}$-terminal amino acid analysis, the sequence of mature pilin, XTLIELM (the first residue produced no reaction presumably because it was the masked amino acid $\mathrm{N}$-methyl-phenylalanine), was obtained. Amino acid analysis of blotted pilin was also carried out to determine the integrity of the carboxyterminal region. The pilin of $P$. aeruginosa 1244 contains six prolines (Castric et al., 1989), mainly in the carboxyterminal region. The observed $M_{\mathrm{r}}$ difference of approximately 1200 , if caused by cleavage in this region, would require breaking the bond between residues 136 and 137 of mature pilin resulting in a change in apparent proline number from six to three. Normalization (with alanine) of actual values obtained gave a proline/alanine $(\mathrm{P} / \mathrm{A})$ ratio of 0.36 , with a P/A ratio of 0.20 predicted for a truncated form and a P/A ratio of 0.35 predicted for mature pilin. Normalization with leucine values gave a proline/leucine $(\mathrm{P} / \mathrm{L})$ ratio of $0.68(\mathrm{a} / \mathrm{P} / \mathrm{L}$ ratio of 0.33 is predicted for a truncated form and a $\mathrm{P} / \mathrm{L}$ ratio of 0.67 for mature pilin). These results suggest that the pilin carboxy-terminal region must be substantially intact.

Purified pili from strains 1244N3(pPAC24) and 1244N3(pPAC46) were treated with periodic acid to oxidize any sugar residues present. These proteins were then treated with digoxigenin-succinyl- $\varepsilon$-amidocaproic acid hydrazide which will form a covalent bond with oxidized sugars. After SDS-PAGE these proteins were electroblotted to nitrocellulose paper and probed with an anti-digoxigenin antibody preparation. Fig. 6 shows that pilin from strain 1244N3(pPAC46) reacted with this antibody indicating the presence of sugar moieties, while pilin from strain 1244N3(pPAC24) gave no reaction.

Pili were dissociated into pilin monomers and dimers in the presence of $30 \mathrm{mM}$ octyl glucoside (Watts et al., 1982a, $\mathrm{b}, 1983$ ), subjected to isoelectric focusing, then blotted to nitrocellulose paper and probed with pilin-specific monoclonal antibodies. Pilin produced by strain 1244N3(pPAC46) or by wild-type strain 1244 (results not shown) had a pI of 4.75 (Fig. 5b). Pilin from strain 1244N3(pPAC24), which lacked a functional pilO gene, had a pI of approximately $6 \cdot 25$. The $\mathrm{pI}$ of mature strain 1244 pilin is predicted from the pil $A$ gene sequence (assuming that the $\mathrm{N}$-terminus is blocked and the two cysteines form a disulfide) to be 7.00. These results indicate that a significant alteration in pilin charge arrangement (neutralization of positive charges or introduction of negative ones) is brought about by the presence of the pilO gene. The difference in charge between these two pilin forms was confirmed by PAGE in the presence of $30 \mathrm{mM}$ octyl glucoside at $\mathrm{pH} 8.8$. Pilin from strain 1244 or strain $1244 \mathrm{~N} 3$ (pPAC46) had a greater net negative charge than pilin from strain 1244N3(pPAC24) (results not shown). Pilin from strains 1244 and $1244 \mathrm{~N} 3$ (pPAC46) also focused at $\mathrm{pH} 4.75$ in the presence of $8.0 \mathrm{M}$ urea and $2.5 \%(\mathrm{v} / \mathrm{v})$ Triton X-100 (results not shown). Pilin preparations from strain 1244N3(pPAC46) or the wild-type strain contained trace amounts of the neutral pilin form (results not shown) possibly due to chemical or enzymic action. The small amounts of the acidic pilin form produced by strain 1244N3(pPAC24) (Fig. 5b), as well as the higher $M_{\mathrm{r}}$ form described above, may be due to low levels of pilO gene expression by the host strain. These results altogether suggest that the $P$. aeruginosa strain 1244 pilO gene is required for a post-translational pilin modification, specifically a glycosylation.

\section{DISCUSSION}

While glycosylation of prokaryotic proteins appears to be primarily restricted to the archaeobacteria (Lechner \& Wieland, 1989), reports have suggested the association of polysaccharide with pili (Armstrong et al., 1981; Robertson et al., 1977). Virji et al. (1993) presented strong evidence (carbohydrate detection of blotted pilin and chemical removal of sugars) that Neisseria meningitidis pilin is glycosylated. Results presented in this paper suggest that pilin from $P$. aeruginosa strain 1244 also is glycosylated. The composition of the pilin-associated material remains to be determined. However, several candidates, including the acidic moieties of the lipopolysaccharide core, alginic acid subunits, as well as other acidic elements or phosphorylated compounds, must be considered. Limitations of SDS-PAGE prevent reliable $M_{\mathrm{r}}$ determinations of glycosylated proteins, a test which must await the use of a more accurate technique such as mass spectrometry. Linkage of glycosylated proteins is usually via the reducing end of an oligosaccharide sugar through an $\mathrm{O}$ or $N$-linkage to the hydroxyl group of serine (or threonine) or the amide group of asparagine (Montreuil $e t$ al., 1986). The residues in the region of $N$-linked moieties have the characteristic consensus sequence N-X-S (or T). While this sequence may be found in the $N$. meningitidis pilin primary structure (Virji et al., 1993), it is absent in $P$. aeruginosa strain 1244 pilin, suggesting the presence of an $N$-linkage, a different sequence specificity, or the utilization of an alternative method of attachment. Clearly much work remains in the characterization of this pilinassociated material.

Previous work (Frost \& Paranchych, 1977; Paranchych et al., 1979) has indicated that pili from $P$. aeruginosa strains 
PAO and PAK contained no sugar residues. Since the primary structure of pili from strain 1244 is distinctive when compared to those of strains PAO and PAK (Castric \& Deal, 1994), pilus modification may represent a strain difference. $P$. aeruginosa strains producing pili antigenically related to those of strain 1244 are common among clinical isolates (Castric \& Deal, 1994). Thus, pilin glycosylation by this bacterium could be useful in clinical identification. Likewise, demonstration of the presence of the pilO gene could be of diagnostic value.

Since the absence of glycosylation seems to have no effect on either the formation of pilus fibres or extension and retraction of these fibres (as measured by bacteriophage sensitivity and twitching motility), the function of this modification is unclear. Because all pilin monomers appear to be modified [this must include both the pilin membrane pool (Watts et al., 1982c) as well as pilus fibres], these glycoproteins must contribute greatly to the overall cell surface negativity. Bacterial avoidance of phagocytosis through inhibition of attachment has been well documented (Densen \& Mandell, 1980). Diminished phagocytosis by neutrophils because of electrostatic repulsion has been demonstrated both by streptococcal $\mathrm{M}$ protein (Fischetti, 1989) and the pili of Neisseria gonorrboeae (Heckels et al., 1976). Such a mechanism could also benefit $P$. aeruginosa when growing saprophytically, where the avoidance of phagocytic amoebae could be an important survival mechanism.

Specific recognition by the pili allows attachment of the pathogen to host cells containing the proper receptors (Baker, 1993; Heckels et al., 1976). The acidic modification might stabilize this attachment through the formation of salt bridges once specific binding had occurred. Alternatively pilin glycosylation may be involved in specific or nonspecific adhesion to the host cell which is independent of pilin protein-mediated attachment. Adhesion studies utilizing $\mathrm{PilO}^{+}$and $\mathrm{PilO}^{-}$variants of $P$. aeruginosa strain 1244 , as well as glycosylated and nonglycosylated pili, will be required to clarify these points.

The genetic evidence presented in this paper relating the presence of pilin glycosylation to a functioning $p i l O$ gene indicates that this process has a specific cellular role. This is strongly supported by the findings that the pilO gene is part of the pil $A$ transcriptional unit. Although the pil $A$ and pilO genes, along with a tRNA ${ }^{\text {thr }}$ gene, are present in the form of an operon, two major transcriptional products are generated (an individual pil $A$ message and a pil $A /$ pilO/tRNA ${ }^{\text {thr }}$ transcript) which are present in a ratio of about 100:1. Such results would not be unexpected as pilin is a major cell protein, while PilO would likely be required in only catalytic or regulatory amounts. The difference in transcription levels could be the result of premature termination of message synthesis or relative instability of the transcript corresponding to the pilO region. The role of translation efficiency should also be considered a factor in differential gene expression since the beginning of the pilO message contains a short loop region which includes the start codon. Such a structure has been suggested to be able to significantly reduce translation (Gold, 1988), and may function to further control expression of a gene coding for a product required in small amounts when it shares a promoter with a gene coding for a product needed in large amounts.

The pilO gene product is predicted to code for a protein with a $M_{\mathrm{r}}$ of 50862 using as start codon the ATG beginning at position 729 (Fig. 1). Hydropathy profile (Kyte \& Doolittle, 1982) of the primary structure indicates that PilO contains nine hydrophobic regions which are flanked by clusters of charged residues. Secondary structure prediction (Chou \& Fassman, 1974) suggests that large portions of these hydrophobic regions are composed of $\beta$-structure which are of adequate length to span the $3.0 \mathrm{~nm}$ membrane lipid core. Positively charged residues flanking the hydrophobic segments could be expected to stabilize this structure through ionic interaction with membrane phospholipids, while charged and polar residues in these regions would promote a surface solvent interaction on both sides of a membrane. Although the location of PilO has not been demonstrated, the high degree of hydrophobicity within the predicted transmembrane regions suggests that it resides in the cytoplasmic membrane. This location would be ideal if PilO functions catalytically on the periplasm side of the cytoplasmic membrane to transfer carrier lipid-bound oligosaccharide subunits to emerging pilin monomers.

\section{ACKNOWLEDGEMENTS}

The author thanks J. C. Sadoff and C. D. Deal for valuable discussion and suggestions. This work was supported by grant AI24118 from the National Institute of Allergy and Infectious Diseases and by US Army grants DAAL03-86-D-0001 and DAAL03-88-G-0103.

\section{REFERENCES}

Armstrong, G. D., Frost, L. S., Vogel, H. J. \& Paranchych, W. (1981). Nature of the carbohydrate and phosphate associated with ColB2 and EDP208 pilin. J Bacteriol 145, 1167-1176.

Baker, N. R. (1993). Mucosal adherence of Pseudomonas aeruginosa. In Pseudomonas aeruginosa, the Opportunist: Pathogenesis and Disease, pp. 7-24. Edited by R. B. Fick. Boca Raton: CRC Press.

Bradley, D. E. (1972). Shortening of Pseudomonas aeruginosa pili after RNA-phage adsorption. J Gen Microbiol 72, 303-319.

Bradley, D. E. (1980). A function of Pseudomonas aeruginosa PAO polar pili: twitching motility. Can J Microbiol 26, 146-154.

Castric, P. A. \& Deal, C. D. (1994). Differentiation of Pseudomonas aeruginosa pili based on sequence and B-cell epitope analysis. Infect Immun 62, 371-376.

Castric, P. A., Sidberry, H. F. \& Sadoff, J. C. (1989). Cloning and sequencing of the Pseudomonas aeruginosa 1244 pilin structural gene. Mol \& Gen Genet 216, 75-80.

Chou, P. Y. \& Fassman, G. D. (1974). Conformational parameters for amino acids in helical, $\beta$-sheet, and random coil regions calculated from proteins. Biochemistry 13, 211-221.

Densen, P. \& Mandell, G. L. (1980). Phagocyte strategy vs. microbial tactics. Rev Infect Dis 2, 817-838.

Elleman, T. C. (1988). Pilins of Bacteroides nodosus: molecular basis of serotypic variation and relationships to other bacterial pilins. Microbiol Rev 52, 233-247. 
Faast, R., Ogierman, M. A., Stroeher, U. W. \& Manning, P. A. (1989). Nucleotide sequence of the structural gene $t c p A$, for a major pilin subunit of Vibrio cholerae. Gene 85, 227-231.

Fischetti, V. A. (1989). Streptococcal M protein: molecular design and biological behavior. Clin Microbiol Rev 2, 285-314.

Frost, L. S. \& Paranchych, W. (1977). Composition and molecular weight of pili purified from Pseudomonas aeruginosa K. J Bacteriol 131, 259-269.

Furste, J. P., Pansegrau, W., Frank, R., Blocker, H., Scholz, P., Bagdasarian, M. \& Lanka, E. (1986). Molecular cloning of the plasmid RP4 primase region in a multi-host-range tac $\mathrm{P}$ expression vector. Gene 48, 119-131.

Gold, L. (1988). Post transcriptional regulatory mechanisms in Escherichia coli. Annu Rev Biocbem 57, 199-233.

Heckels, J. E., Blackett, B., Everson, J. S. \& Ward, M. E. (1976). The influence of surface charge on the attachment of Neisseria gonorrboeae to human cells. $J$ Gen Microbiol 76, 359-364.

Henrichsen, J. (1983). Twitching motility. Annu Rev Microbiol 37, 81-93.

Hobbs, M., Dalrymple, B., Delaney, S. F. \& Mattick, J. S. (1988). Transcription of the fimbrial subunit gene and an associated transfer RNA gene of Pseudomonas aeruginosa. Gene 62, 219-227.

Johnson, K., Parker, M. L. \& Lory, S. (1986). Nucleotide sequence and transcriptional initiation site of two Pseudomonas aeruginosa pilin genes. J Biol Chem 261, 15703-15708.

Kyte, J. \& Doolittle, R. F. (1982). A simple method for displaying the hydropathic character of a protein. J Mol Biol 157, 105-132.

Lechner, J. \& Wieland, F. (1989). Structure and biosynthesis of procaryotic glycoproteins. Annu Rev Biocbem 58, 173-194.

Marrs, C. F., Schoolnik, G., Koomey, J. M., Hardy, J., Rothbard, J. \& Falkow, S. (1985). Cloning and sequencing of a Moraxella bovis pilin gene. J Bacteriol 163, 132-139.

Meyer, T. F., Billyard, E., Haas, R., Storzbach, S. \& So, M. (1984). Pilus genes of Neisseria gonorrbeae: chromosomal organization and DNA sequence. Proc Natl Acad Sci USA 81, 6110-6114.

Montreuil, J., Bouquelet, S., Debray, H., Fournet, B., Spit, G. \& Strecker, G. (1986). Glycoproteins. In Carbobydrate Analysis, a Practical Approach, pp. 143-204. Edited by M. F. Chaplin \& J. F. Kennedy. Oxford: IRL Press.

Ottow, J. C. G. (1975). Ecology, physiology, and genetics of fimbriae and pili. Annu Rev Microbiol 29, 79-108.

Paranchych, W. \& Frost, L. S. (1988). The physiology and biochemistry of pili. Adv Microb Physiol 29, 53-114.

Paranchych, W., Sastry, P. A., Frost, L. S., Carpenter, M., Armstrong, G. D. \& Watts, T. H. (1979). Biochemical studies on pili isolated from Pseudomonas aeruginosa strain PAO. Can J Microbiol 25, 1175-1181.

Ramphal, R., Sadoff, J. C., Pyle, M. \& Silipigni, J. D. (1984). Role of pili in the adherence of Pseudomonas aeruginosa to injured tracheal epithelium. Infect Immun 44, 38-40.

Ramphal, R., Koo, L., Ishimoto, K. S., Totten, P. A., Lara, J. C. \& Lory, S. (1991). Adhesion of Pseudomonas aeruginosa pilin-deficient mutants to mucin. Infect Immun 59, 1307-1311.
Rao, V. K. \& Progulske-Fox, A. (1993). Cloning and sequencing of two type 4 ( $N$-methylphenylalanine) pilin genes from Eikenella corrodens. J Gen Microbiol 139, 651-660.

Reddy, K. J. (1989). Preparation of bacterial RNA. In Current Protocols in Molecular Biology, vol. 1, 4.4.1.-4.4.7. Edited by F. M. Ausubel, R. Brent, R. E. Kingston, D. D. Moore, J. G. Seidman, J. A. Smith \& K. Struhl. New York: John Wiley.

Robertson, J. N., Vincent, P. \& Ward, M. E. (1977). The preparation and properties of gonococcal pili. J Gen Microbiol 102, 169-177.

Ruvkun, G. B. \& Ausubel, F. M. (1981). A general method for sitedirected mutagenesis in prokaryotes. Nature 289, 65-88.

Saiman, L., Sadoff, J. \& Prince, A. (1989). Cross-reactivity of Pseudomonas aeruginosa antipilin monoclonal antibodies with heterogeneous strains of $P$. aeruginosa and Pseudomonas cepacia. Infect Immun 57, 2764-2770.

Sambrook, J., Fritsch, E. F. \& Maniatis, T. (1989). Molecular Cloning: a Laboratory Manual, vol. 1, 2nd edn. Cold Spring Harbor, NY: Cold Spring Harbor Laboratory.

Sanger, F., Nicklen, S. \& Coulson, A. R. (1977). DNA sequencing with chain terminating inhibitors. Proc Natl Acad Sci USA 74, 5463-5467.

Silipigni-Fusco, J. D. (1987). Studies on the role of somatic pili as virulence and immunity factors in the pathogenicity of Pseudomonas aeruginosa. PhD thesis, University of Pittsburgh, USA.

Virji, M., Saunders, J. R., Sims, G., Makepeace, K., Maskell, D. \& Ferguson, D. J. P. (1993). Pilus-facilitated adherence of Neisseria meningitidis to human epithelial and endothelial cells: modulation of adherence phenotype occurs concurrently with changes in primary amino acid sequence and the glycosylation status of pilin. Mol Microbiol 10, 1013-1028.

Watts, T. H., Kay, C. M. \& Paranchych, W. (1982a). Dissociation and characterization of pilin isolated from Pseudomonas aeruginosa strains PAK and PAO. Can J Biocbem 60, 867-872.

Watts, T. H., Scraba, D. G. \& Paranchych, W. (1982b). Formation of 9-nm filaments from pilin monomers obtained by octyl-glucoside dissociation of Pseudomonas aeruginosa pili. J Bacteriol 151, 1508-1513.

Watts, T. H., Worobec, E. A. \& Paranchych, W. (1982c). Identification of pilin pools in the membranes of Pseudomonas aeruginosa. $J$ Bacteriol 152, 687-691.

Watts, T. H., Kay, C. M. \& Paranchych, W. (1983). Spectral properties of three quaternary arrangements of Pseudomonas pilin. Biocbemistry 22, 3640-3646.

Weir, S. \& Marrs, C. F. (1992). Identification of type 4 pili in Kingella denitrificans. Infect Immun 60, 3437-3441.

West, S. E. H. \& Iglewski, B. H. (1988). Codon usage in Pseudomonas aeruginosa. Nucleic Acids Res 16, 9323-9335.

Wood, D. E., Strauss, D. C., Johanson, W. G., Berry, V. K. \& Bass, J. A. (1980). Role of pili in adherence of Pseudomonas aeruginosa to mammalian buccal epithelial cells. Infect Immun 29, 1146-1151.

Received 2 September 1994; revised 19 January 1995; accepted 2 February 1995. 